\title{
A INFLUÊNCIA DA MÍDIA NOS ATENDIMENTOS CARDIOLÓGICOS DE URGÊNCIA: 0 CASO BUSSUNDA
}

\author{
Juliana Sobreira Taberner*, Pedro Colli Badino de Souza leite, Martin Mendonça Cutait, Julio Takada, Antônio de Pádua Mansur, \\ Bruno Caramell \\ Trabalho realizado no Instituto do Coração do Hospital das Clínicas da Faculdade de Medicina da Universidade de São Paulo
}

\author{
* Correspondência \\ Rua Oscar Freire, 1549, ap.34 \\ São Paulo - SP \\ jutaberner@yahoo.com.br
}

\section{RESUMO}

Convulsões sociais associadas a catástrofes como enchentes, terremotos e ataques aéreos apresentam maior incidência de infarto do miocárdio. A morte prematura de personagens da mídia com forte apelo popular podem representar fenômenos semelhantes.

Oвjetıvos. Analisar o impacto da mídia sobre o serviço de atendimento de emergência do InCor.

Métodos. Através do banco de dados do InCor, foi analisado o perfil demográfico dos pacientes atendidos nos dias subseqüentes às repercussões na imprensa leiga da morte por infarto agudo do miocárdio de um importante personagem da mídia (Grupo Bussunda). Esses pacientes foram comparados com um Grupo Controle representado pelos atendimentos no primeiro semestre de 2006.

Resultados. Comparado ao Grupo Controle, o grupo Bussunda (Grupo B) apresentou mudanças que foram estatisticamente significativas e foram representadas pela redução da faixa etária e pelo aumento do número de homens atendidos em relação a mulheres. Este achado refletiu uma aproximação do primeiro grupo com o perfil do personagem em questão (homem, 43 anos de idade). Estas mudanças persistiram por quatro dias em relação à idade e por um dia em relação ao gênero. Apesar do aumento absoluto dos atendimentos, não ocorreu aumento do número de óbitos e de internações por infarto do miocárdio.

Conclusão. A divulgação pela mídia das circunstâncias e da morte prematura por infarto agudo do miocárdio de um personagem importante provocou aumento do número de atendimentos e alterações do perfil demográfico dos pacientes que procuram o serviço de emergência cardiológica, sem que houvesse interferência no número de óbitos ou de internações.

Unitermos: Infarto do miocárdio. Pronto-socorro. Emergência. Mídia.

\section{INTRODUÇÃO}

A relação entre a mídia e a saúde tem sido objeto de debate na literatura. Tanto de um lado, valorizando demasiadamente resultados sem significado médico, quanto de outro, divulgando tratamentos sem comprovação científica ${ }^{1-2}$. A ocorrência de um aumento de atendimentos por queixas cardiológicas foi um fenômeno citado freqüentemente na imprensa leiga (veiculado a cerca de 22 milhões de indivíduos) quando da morte de um importante personagem da televisão $0^{3-4}$. Entretanto, um estudo com dados concretos associados a uma análise estatística adequada e a uma interpretação dos mecanismos envolvidos ainda não foi publicado. Este estudo teve por objetivo analisar as características do atendimento cardiológico de urgência no maior pronto-socorro especializado em cardiologia do país no período próximo à morte de um personagem da mídia televisiva e compará-lo aos períodos anteriores.

\section{Métodos}

Foi realizado um estudo transversal retrospectivo utilizando o banco de dados do Instituto do Coração (InCor) do Hospital das Clínicas da Faculdade de Medicina da Universidade de São Paulo. Foram coletadas informações referentes aos indivíduos atendidos no pronto-socorro da Instituição durante o primeiro semestre do ano de 2006. O InCor caracteriza-se por ser um instituto integrante do complexo Hospitalar Terciário e Universitário, que é exclusivamente destinado ao atendimento de doenças cardiovasculares. 0 pronto-socorro do InCor atende pacientes que o procuram espontaneamente ou que são encaminhados de outros serviços médicos. Durante o período de $1^{\circ}$ de janeiro a 31 de julho de 2006 foram atendidas em média I 55 pessoas por dia, num total de 32.876 indivíduos. Desta população, foram excluídos aqueles com inconsistência de dados (dados faltantes, gênero e idade preenchidos inadequadamente), os menores de 18 anos e aqueles com mais de 96 anos de idade, assim como as repetições. A amostra final selecionada para o estudo foi de 31.018 indivíduos.

A data de 19 de junho de 2006 foi selecionada por representar o primeiro dia útil subseqüente às repercussões na imprensa leiga da morte por infarto do miocárdio de um importante personagem da mídia do país ${ }^{3-4}$. As variáveis de interesse coletadas foram: número de atendimentos, idade, gênero, destino dado ao paciente (internação ou alta hospitalar) e queixa principal. Posteriormente, foi realizada uma análise descritiva das variáveis supracitadas relacionadas aos atendimentos do mês de junho de 2006. A análise visual gráfica permitiu identificar o período de 19 a 23 de junho como sendo aquele onde o número de atendimentos aumentou (Grupo Bussunda) - Figura I. A seguir, foram analisadas as variáveis de 


\section{Figura I -Atendimentos, Obitos e Internaçóes no Serviço de}

Emergência do InCor em junho/2006

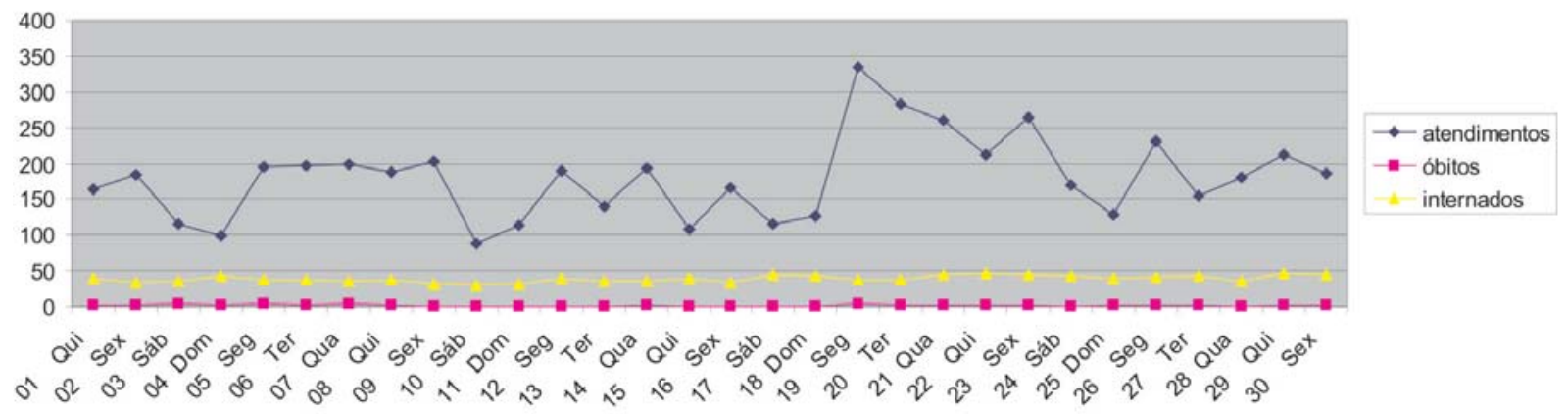

interesse de cada um dos dias do intervalo selecionado e os valores foram expressos em médias e desvios-padrão. Os parâmetros relativos a cada dia foram comparados aos valores referentes ao conjunto do período de $1^{\circ}$ de janeiro a 31 de julho de 2006, excluindo o dia selecionado (Grupo Controle).

A análise comparativa da idade foi realizada por meio do teste t não pareado. Para a comparação entre os gêneros foi utilizado o teste do Qui-quadrado. O nível de significância estatística adotado foi o de $p<0,05$.

\section{Resultados}

No período analisado, correspondente ao primeiro semestre de 2006, 31.018 indivíduos foram selecionados para o estudo, sendo $54,6 \%$ do gênero feminino e $45,4 \%$ do masculino. A distribuição dos atendimentos durante o mês de junho de 2006 encontra-se representada na Figura I. Na Tabela I está representada a distribuiçãa por genêros dos indivíduos atendidos durante o período de 19 a 23 de junho que representa aquele onde o número de atendimentos aumentou (Grupo Bussunda) em comparação ao Grupo Controle (todo o semestre analisado).

A análise do intervalo entre 19 e 23 de junho de 2006 demonstra uma alteração do perfil demográfico do Grupo Bussunda em relação ao Grupo Controle. Essa variação foi observada em relaçãa à idade e ao gênero dos indivíduos estudados.

No tocante à idade, foi verificada uma redução estatisticamente significativa da média etária dos indivíduos do Grupo Bussunda. Essa redução se manteve durante quatro dias após o evento estudado, como mostra a Figura 2.

No corte transversal do dia 19 de junho de 2006, data imediatamente posterior ao evento estudado, foi aferida uma inversão no perfil dos gêneros. Contudo, essa inversão só pôde ser vista nesse dia, sendo que a partir da data 20 de junho de 2006 retorna o predomínio de atendimentos do gênero feminino.

\section{Discussão}

O presente estudo revelou que o perfil da população atendida no pronto-socorro do InCor sofreu modificações no período entre

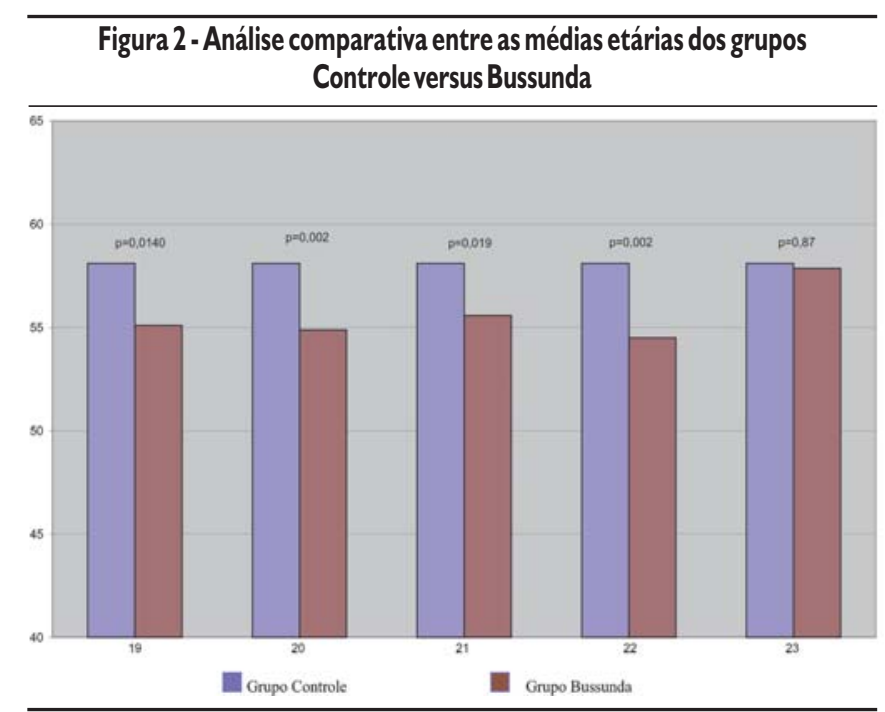

Tabela I- Porcentagem de atendimentos de homens e mulheres nos cinco dias após o evento estudado e durante todo semestre analisado

\begin{tabular}{|c|c|c|c|c|c|c|}
\hline & 19/06 & $20 / 06$ & $21 / 06$ & $22 / 06$ & $23 / 06$ & Controle \\
\hline $\begin{array}{l}\text { \% Homens } \\
\text { \% Mulheres } \\
\text { Valordep }\end{array}$ & $\begin{array}{l}52,3 \\
47,7 \\
0,01\end{array}$ & $\begin{array}{l}47,8 \\
52,2 \\
0,39\end{array}$ & $\begin{array}{l}43,7 \\
56,3 \\
0,62\end{array}$ & $\begin{array}{l}40,5 \\
59,5 \\
0,18\end{array}$ & $\begin{array}{l}43,3 \\
56,7 \\
0,45\end{array}$ & $\begin{array}{l}45 \\
55\end{array}$ \\
\hline
\end{tabular}

19 e 23 de junho de 2006. Estas modificações foram caracterizadas pela mudança no número e nas características demográficas de indivíduos que foram atendidos. Estudos anteriores sugeriram que situações de intenso estresse emocional como terremotos ou bombardeios aéreos estão associados a um aumento na incidência de internações e de óbitos por síndromes coronarianas agudas ${ }^{5-8}$. Em nosso estudo, observamos um aumento no número de atendimentos em um pronto-socorro de cardiologia que foi associado à divulgação, pela imprensa, de um problema cardíaco grave que levou à morte de um personagem público. $\mathrm{O}$ aumento de atendimentos por sintomas cardiológicos pode estar relacionado à 
identificação da população com o perfil do personagem vítima de um infarto ou com a divulgação em nível nacional dos sintomas da doença coronariana aguda. Os achados de nosso estudo confirmam esta hipótese uma vez que não observamos aumento paralelo do número de internações ou de diagnósticos de infarto do miocárdio, ao contrário dos estudos que analisaram catástrofes como bombardeios e terremotos onde ocorreu aumento real da incidência da doença (maior número de internações) e de óbitos a ela relacionados.

A percepção de que a doença coronariana tem um curso clínico silencioso e que pode ter como primeira manifestação um evento fatal, pode ter provocado medo na população geral. Isso levou à grande procura do serviço de emergência do InCor, assim como provavelmente em todo o país. O aumento do número de atendimentos realizados para indivíduos do sexo masculino e de faixa etária mais jovem fortalece esta idéia em função de uma provável identificação com o personagem da mídia (43 anos e sexo masculino).

A identificação deste fenômeno pode permitir a elaboração de estratégias de prevenção primária da doença cardiovascular por meio de ampla divulgação dos fatores de risco e da necessidade de seu controle.

\section{Conclusão}

A divulgação pela imprensa leiga dos sinais e sintomas que precederam a morte por infarto do miocárdio de importante personagem da mídia brasileira associou-se à modificação efêmera do perfil demográfico dos indivíduos atendidos em pronto-socorro cardiológico, sem conseqüências para mortalidade ou freqüência de internações. Exemplos como este salientam a importância da necessidade da otimização da relação entre a mídia e o sistema de saúde, envolvendo jornalistas e médicos. Esta relaçãa, se mais bem explorada, pode permitir o conhecimento do comportamento da população e a elaboração de estratégias adicionais para prevenção cardiovascular.

\section{Conflito de interesse: não há.}

\section{SUMMARY}

\section{IMPACT OF MEDIA COVERAGE ON CARDIAC EMERGENCY CARE : THE BUSSUNDA CASE}

INTRODUCTION: Social turmoil associated with catastrophes such as flooding, earthquakes or air raids bring about an increased incidence of acute myocardial infarction. Premature death of media personalities with a strong public appeal may cause similar phenomena.

OBIECTINE: To analyze the impact of this media coverage on the emergency service at a Heart Hospital in Brazil (InCor).

METHODS: Demographic profiles of patients collected from the InCor's database were analyzed in the days following the death of an important TV personality (Group B). These patients were compared to a Control Group represented by those attended during the first semester of 2006.

Results: When compared to the Control Group, Group B disclosed the following significant statistical changes: patients were younger and more of them were male. These findings reflect a change of profile in the control group tending to a greater similarity with the characteristics of the TV personality (man, 43 years old). These changes remained significant for four days (age) and one day (gender). Furthermore, the greater demand for the cardiologic emergency service was not followed by an increased number of deaths and hospitalizations for acute myocardial infarction.

CONCluSION: Media exposure on the circumstances of premature death by myocardial infarction of an important TV personality was associated with an increase in the number and a change in the profile of the patients seeking the emergency room at the InCor Heart Hospital. Differently from a situation such as catastrophes, this phenomenon was not followed by an increase in the incidence of myocardial infarction [Rev Assoc Med Bras 2007; 53(4): 335-7]

KEY wORDS: Myocardial infarction. Media. Emergency service

\section{REFERÊNCIAS}

I. Singh J, Hallmayer J, Illes J. Interaction and paradoxal forces in neuroscience and society. Nat Rev Neurosci. 2007;8:I53-9.

2. Praça JM, Thomaz A, Caramelli B. Eggplant (Solanum melongena) extract does not alter serum lipid levels. Arq Bras Cardiol. 2004;82(3):269-76;

3. Edição do Fantástico 2006 Jun. Os fatores de risco de um ataque cardíaco [citado 5 dez 2006]. Disponível em: http:// fantastico.globo.com/Jornalismo/Fantastico/0, ,AA I 2 1 9026-40050-0- | 8062006,00.html.

4. Edição da Rede Globo de Televisão 2006 Jul. Edição Geral de Comercialização [citado jul 2006]. Disponível em: http:// comercial.redeglobo.com.br/programacao_show/fant3_intro.php.

5. Tsai CH, Lung FW, Wang SY. The 1999 ji-ji (Taiwan) earthquake as a trigger for acute myocardial infarction. Psychosomatics. 2004;45(6):477-82

6. Zubaid M, Suresh CG, Thalib L, Rashed W. Could missile attacks trigger acute myocardial infarction? Acta Cardiol. 2006;6I(4):427-3I.

7. Rotenberg Z, Noy S, Gabbay U. Israeli ED experience during the Gulf War. Am J Emerg Méd. 1994; 12(1): I 18-9.

8. Ogawa K, Tsuji I, Shiono K, Hisamichi S. Increased acute myocardial infarction mortality following the 1995 Great Hanshin-Awaji earthquake in Japan. Int J Epidemiol. 2000;29(3):449-55.

Artigo recebido: 29/1 I/06

Aceito para publicação: 07/05/07 